UCRL-JC-125841

PREPRINT

\title{
Effect of Enhanced Thermal Dissipation on the Rayleigh-Taylor Instability in Emulsion-Like Media
}

\author{
A. Toor \\ D. Ryutov
}

This paper was prepared for submittal to the 4th International Conference on Dense Z-Pinches Vancouver, B.C., Canada

May 23-30, 1997

July 3, 1997

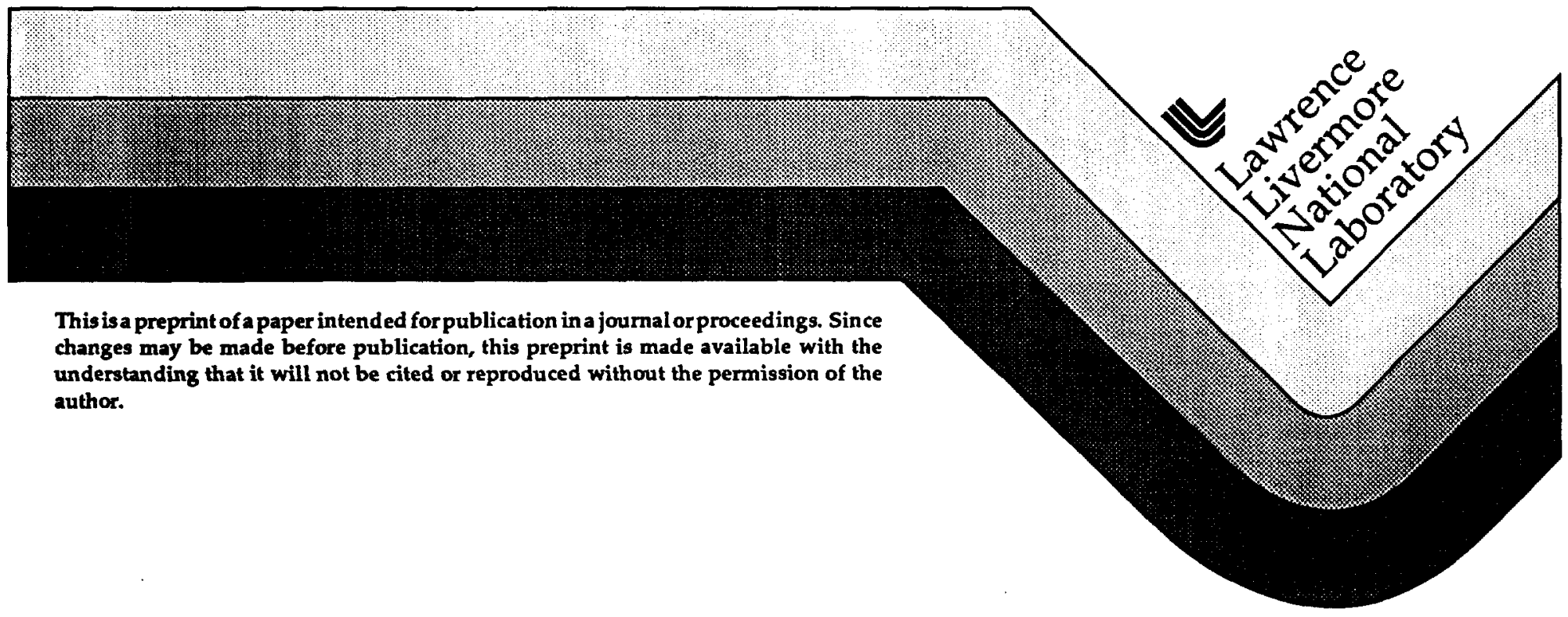




\section{DISCLAMER}

This document was prepared as an account of work sponsored by an agency of the United States Government. Neither the United States Government nor the University of California nor any of their employees, makes any warranty, express or implied, or assumes any legal liability or responsibility for the accuracy, completeness, or usefulness of any information, apparatus, product, or process disclosed, or represents that its use would not infringe privately owned rights. Reference herein to any specific commercial product, process, or service by trade name, trademark, manufacturer, or otherwise, does not necessarily constitute or imply its endorsement, recommendation, or favoring by the United States Government or the University of California. The views and opinions of authors expressed herein do not necessarily state or reflect those of the United States Govemment or the University of California, and shall not be used for advertising or product endorsement purposes. 


\title{
Effect of Enhanced Thermal Dissipation on the Rayleigh-Taylor Instability in Emulsion-Like Media
}

\author{
A. Toor, D. Ryutov, \\ Lawrence Livermore National Laboratory, Livermore, CA 94551, USA
}

\begin{abstract}
Rayleigh-Taylor instability in a finely structured emulsion-like medium consisting of the two components of different compressibility is considered. Although the term "emulsion" is used to describe the structure of the medium, under typical fast Z-pinch conditions both components behave as gases. The two components are chosen in such a way that their densities in the unperturbed state are approximately equal. Specific emphasis has been made on the analysis of perturbations with the scale $\lambda$ considerably exceeding the size of the grains $a$. Averaged equations describing such perturbations are derived. The difference in compressibility of the two components leads to the formation of temperature variations at the scale $a$, and increases the rate of the thermal dissipation by a factor $(\mathrm{Na})^{2}$. The strongest stabilizing effect of the thermal dissipation takes place when the thermal relaxation time is comparable with the instability growth rate.
\end{abstract}

In this paper we consider a phenomenon that may be helpful in mitigating the Rayleigh-Taylor instability of imploding liners, namely a phenomenon of enhanced thermal dissipation in emulsion-like media. We assume that the fluid (or gas) is twocomponent, with fine grains of one component randomly, but on the average uniformly, distributed in the other component. With respect to motions with the scale $\lambda$ considerably exceeding the scale of the fine structure, $a$, this fluid behaves more or less as a uniform fluid, with some average density and average thermodynamic functions. In particular, it may have a gradient of the average density that would drive the Rayleigh-Taylor instability. We use the word "emulsion" in a somewhat loose sense, just because its visual image is close to the structures we are studying. In fusion-related and pulse-power applications, the initial state will most probably be that of a heterogeneous solid. In fusion-related experiments, this solid usually experiences fast heating and becomes a gas (inhomogeneous in our case) early in the pulse. For this reason, all our analysis will be based on the hydrodynamics equations, without accounting for elastic forces.

Our approach to the mitigation of the Rayleigh-Taylor instability is based on the observation that the presence of a fine internal scale may considerably enhance the dissipation rate (Cf. [1]). The origin of the enhanced dissipation is as follows: the two components that form the emulsion, have, generally speaking, different compressibilities. Therefore, when the pressure perturbation associated with the Rayleigh-Taylor instability develops, the temperature perturbation becomes unequal in the two components. Then the thermal dissipation begins, but at the scale $a$ which is much smaller than the scale $\lambda$ of the perturbation; accordingly the dissipation rate becomes $(\mathrm{N} / \mathrm{a})^{2}$ times higher than for a homogeneous fluid. Clearly, the justdiscussed mechanism works only in compressible media.

In the case of wire arrays, it might be simpler to produce a kind of $2 \mathrm{D}$ emulsion. The simplest way would be to use the wire array where the neighboring wires would be made of different materials (Fig. 1a). This would produce a "coarsegrained" emulsion. A more sophisticated way would be to use bunches of wires of different composition, possibly interwoven within every bunch. The whole array

" On leave from Budker Institute of Nuclear Physics, Novosibirsk 630090, Russia. 
would then be assembled of a large number of such bunches (Fig. 1b). After evaporation at early stages of the discharge, separate conductors gradually merge and form a continuous shell of the liner, but the initially embedded non-uniformities should survive this process because of a very low diffusion coefficient. Of issue may be the convective mixing of the two components. However, one should expect that under optimum conditions, where the instabilities are kept under control, there won't be too violent convective motions in the liner.

One can expect that the maximum dissipation will occur if the grain size is chosen in such a way that the characteristic heat exchange time between the grains,

$$
\tau \sim a^{2} / \chi
$$

(where $\chi$ is thermal diffusivity) is of the order of a time-scale $\omega^{-1}$ of development of the instability. Indeed, if compression (rarefaction) occurs too rapidly, so that heat exchange between the neighboring grains doesn't have time to develop, the process is purely adiabatic and dissipation is absent. On the contrary, if the change of the volume occurs too slowly, the temperature remains uniform and dissipation vanishes again. The compressibilities of the medium, $\delta \rho / \delta p$, in these two limiting cases are different: in the first case the compressibility is adiabatic, in the second case it is isothermal. By $\delta p$ and $\delta \rho$ we mean perturbations of the pressure and of the density of macroscopic volumes, i.e., the volumes containing many grains. One has:

$$
(\delta \rho / \delta p)_{\text {fast }}=1 / s_{\text {fast }}^{2} ;(\delta \rho / \delta p)_{\text {slow }}=1 / s_{\text {slow }}^{2}
$$

where $s$ is a sound speed for the respective process $\left(s_{\text {fast }}>s_{\text {slow }}\right)$.

As was shown in Ref. [2], one can use the following interpolation that covers intermediate frequencies (where thermal dissipation is important):

$$
\delta p_{L}=\hat{s}^{2} \delta \rho_{L} ; \hat{s}^{2} \equiv F(\omega) s_{\text {slow }}^{2}
$$

where

$$
F(\omega)=\frac{1-i \omega \tau\left(s_{\text {fast }} / s_{\text {slow }}\right)^{2}}{1-i \omega \tau}
$$

The subscript " $L$ " in Eq. (3) shows that $\delta p$ and $\delta \rho$ are Lagrangian perturbations, related to a particular element of the fluid. The Lagrangian and Eulerian perturbations are related to each other in a standard way:

$$
\delta \rho=-\xi \cdot \nabla \rho+\delta \rho_{L} ; \delta p=-\xi \cdot \nabla p+\delta p_{L},
$$

with $\delta \rho_{L}=-\rho \nabla \cdot \xi$, and $\xi$ being a displacement of a certain macroscopic element with respect to its unperturbed position.

In the stability analysis, we assume that the gravity acceleration is directed downward along the $z$ axis, $g_{z} \equiv-g$, with $g>0$. Equilibrium pressure distribution obeys the barometric law:

$$
p^{\prime}=-\rho g \equiv-p / h
$$

where the prime designates the $z$-derivative, and $h$ is a scale-length of the unperturbed pressure and density variation. We consider perturbations of the form $f(z) \exp (-i \omega t+i k x)$ and assume that the scale-length of the perturbations satisfies condition $t>>a$. In this case, the basic set of equations describing small perturbations reads as: 


$$
\begin{aligned}
& \omega^{2} \rho \xi_{x}=i k \delta p \\
& \omega^{2} \rho \xi_{z}=\delta p^{\prime}+g \delta \rho, \\
& \delta \rho=-\rho\left(i k \xi_{x}+\xi_{z}^{\prime}\right)-\xi_{z} \rho^{\prime}, \\
& \delta p=-p^{\prime} \xi_{z}-F(\omega) \rho s_{s}^{2}\left(i k \xi_{x}+\xi_{z}^{\prime}\right) .
\end{aligned}
$$

All the quantities entering Egs. (6)-(10) are volume-averaged over a scale much greater than $a$ but much smaller than $\lambda$. There is a subtlety at this point: although the densities of the two components are equal in the unperturbed state, they become unequal in the perturbations. The gravity force causes then mutual displacements of the elements of different density, and displacement $\xi$ that enters Eqs. (7)-(10) should be understood as the average over these elements. However, as shown in Ref. [2], in the typical situation, mutual displacements of the two components are insignificant, and a simple interpretation of $\xi$ as a displacement of the whole macroscopic volume remains essentially correct.

Consider stability of localized modes, with the wave-length much less than the scale-length $h$ of the unperturbed state. For such modes one can use the eikonal approximation in the $z$ direction by taking the $z$ dependence of the unknown functions in the form $\exp (i q z)$, where $q$ is the wave number of the perturbation in the $z$ direction. For the fastest growing modes, one has $k>>q$ (see, e.g., [3]). For such modes, the dispersion relation reads as:

$$
\omega^{2}=-g\left(\frac{\rho^{\prime}}{\rho}-\frac{1}{F(\omega)} \frac{p^{\prime}}{\rho s_{s l o w}^{2}}\right) \text {. }
$$

At this point, it is convenient to introduce, instead of the complex frequency $\omega$, the complex growth rate $\Gamma, \Gamma=-i \omega, \operatorname{Re} \Gamma>0$ corresponds to an instability.

It is convenient to present the resulting dispersion relation in the dimensionless form:

where

$$
\tilde{\Gamma}^{2}=1+\frac{\eta}{1+\tilde{\Gamma} \tilde{\tau}}
$$

$$
\tilde{\Gamma}=\frac{\Gamma}{\Gamma_{\text {fast }}} ; \tilde{\tau}=\Gamma_{\text {fast }} \tau\left(\frac{s_{\text {fast }}}{s_{\text {slow }}}\right)^{2} ; \eta=\left(\frac{\Gamma_{\text {slow }}}{\Gamma_{\text {fast }}}\right)^{2}-1 \text {, }
$$

and

$$
\Gamma_{\text {fast }}^{2}=g \frac{\rho^{\prime}}{\rho}+\frac{g^{2}}{s_{\text {fast }}^{2}} ; \Gamma_{\text {slow }}^{2}=g \frac{\rho^{\prime}}{\rho}+\frac{g^{2}}{s_{\text {slow }}^{2}}
$$

When switching from Eq. (11) to (12), we used the equilibrium condition (6). The growth rate $\Gamma_{\text {fast }}$ corresponds to fast (purely adiabatic) perturbations, for which $\tau$ can be considered infinite. The growth rate $\Gamma_{\text {slow }}$ corresponds to slow perturbations, for which $\tau$ can be considered zero. We imply that both $\Gamma_{\text {fars }}^{2}$ and $\Gamma_{\text {slow }}^{2}$ are positive (i.e., the system is unstable with respect to both slow and fast perturbations). Note that $\Gamma_{\text {slow }}>\Gamma_{\text {fast }}$. 
The solutions of the dimensionless dispersion relation (12) vs. the dimensionless relaxation time are presented in Fig. 2. The effect of the thermal relaxation processes on the unstable root becomes noticeable at $\eta$ 's exceeding a few tenths. Note that, in a broad range of the dimensionless relaxation times $\tilde{\tau}$, there appears a mode of an oscillatory damping $(\operatorname{Re} \omega=-\operatorname{Im} \Gamma \neq 0)$. The presence of this mode may considerably change the nonlinear behavior of the system leading to a slower nonlinear growth (because additional - and damped -- degree of freedom appears in the system). Analysis of this part of the problem goes beyond the scope of the present paper. Note also that the appearance of this new mode is a very robust phenomenon: it exists in a broad range of relaxation times, even if the difference of slow and fast compressibilities (characterized by the parameter $\eta$ ) is as low as $5 \%$.

At higher $\tau$ 's, there appears a weakly damped mode (the A-B branch of the dispersion curve in Fig. 2). This mode should also have a stabilizing effect at the nonlinear stage.

\section{ACKNOWLEDGMENTS}

The authors are grateful to Drs. J. De Groot, J. Hammer, P. Springer and P. Wheeler for valuable comments. This work was performed under the auspices of the U.S. Department of Energy by Lawrence Livermore National Laboratory under Contract W7405-ENG-48.

\section{REFERENCES}

1. Landau, L.D., Lifshitz, E.M., Theory of Elasticity. Pergamon Press, 1986.

2. Ryutov, D., Toor, A. "Enhanced Thermal Dissipation and the Rayleigh-Taylor Instability in Emulsion-Like Media". LLNL Report UCRL-JC-127007, 1997 (submitted to Phys. Plasmas).

3. Kull, H.J., Physics Reports, 206, 197 (1991)
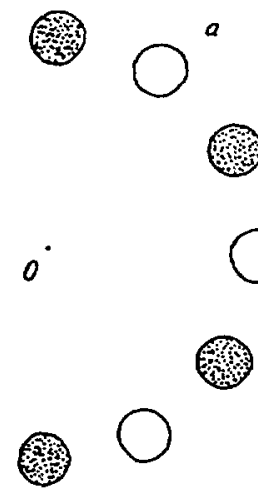

Fig.1 Wire arrays consisting of the wires of two different materials (a) and a wire array consisting of many bunches of interwoven thin wires of two materials (b); $O$ is the axis of the array.



Fig.2 Solutions of the dispersion relation (12): $\eta=0$ (dashed lines); $\eta=0.25$ (solid lines). In the latter case there appears a broad interval of relaxation times $(0.53<\tau<2.1)$ where $\operatorname{Im} \Gamma \neq 0(\operatorname{Im} \Gamma$ is shown in the dotted line). Note the presence of weakly damped mode at large $\tau$ 's (the segment $A B$ ) 


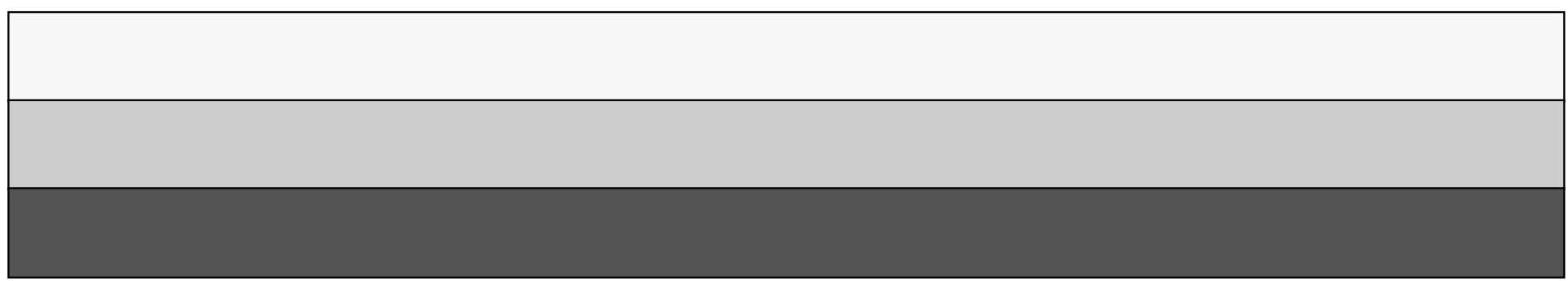

